INPLASY

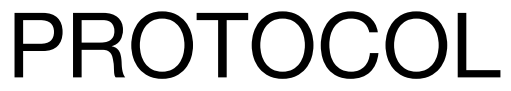

To cite: Zhao et al. Effects of Aerobic Exercise on TC, HDL$\mathrm{C}$, LDL-C and TG in patients with hyperlipidemia : A protocol of Systematic Review and Meta-Analysis. Inplasy protocol 202120037. doi: 10.37766/inplasy2021.2.0037

Received: 10 February 2021

Published: 10 February 2021

Corresponding author: Shaoping Zhao

zhaosp2623@163.com

Author Affiliation:

Department of Teaching and Research of Physical Education, Wuhan

Polytechnic University

Support: whpu-2020-kj-270.

Review Stage at time of this submission: Risk of bias assessment.

Conflicts of interest:

None declared.

\section{Effects of Aerobic Exercise on TC, HDL-C, LDL-C and TG in patients with hyperlipidemia : A protocol of Systematic Review and Meta-Analysis}

Zhao, SP1; Zhong, J2; Sun, $\mathrm{CH}^{3}$; Zhang, JP4 .

Review question / Objective: Participants: Serum total cholesterol $(\mathrm{TC}) \geq 5.20 \mathrm{mmol} / \mathrm{L}$, triglyceride $(\mathrm{TG}) \geq 1.7 \mathrm{mmol} / \mathrm{L}$, high-density lipoprotein cholesterol (HDL-C) $\leq 1.04 \mathrm{mmol} / \mathrm{L}$, low-density lipoprotein cholesterol (LDL-C) $\geq 3.61 \mathrm{mmol} / \mathrm{L}$, no major disease, no blood lipid-lowering drugs in the past six months. Intervention: In the experimental group, all patients must perform aerobic exercises, including walking, cycling, strength training, taiji, yoga, etc. Comparator: The control group did not implement intervention, and did not receive other hypolipidemic treatments. Outcomes: Primary outcomes: The primary outcomes are mainly evaluated by total cholesterol and triglycerides. Secondary outcome: The secondary outcomes are assessed by HDL-C, LDL-CI, ApoA1 and ApoB indexes.

INPLASY registration number: This protocol was registered with the International Platform of Registered Systematic Review and Meta-Analysis Protocols (INPLASY) on 10 February 2021 and was last updated on 10 February 2021 (registration number INPLASY202120037).

\section{INTRODUCTION}

Review question / Objective: Participants: Serum total cholesterol (TC) $\geq 5.20 \mathrm{mmol} / \mathrm{L}$, triglyceride (TG) $\geq 1.7 \mathrm{mmol} / \mathrm{L}$, high-density lipoprotein cholesterol (HDL-C) $\leq 1.04$ $\mathrm{mmol} / \mathrm{L}$, low-density lipoprotein cholesterol
(LDL-C) $\geq 3.61 \mathrm{mmol} / \mathrm{L}$, no major disease, no blood lipid-lowering drugs in the past six months. Intervention: In the experimental group, all patients must perform aerobic exercises, including walking, cycling, strength training, taiji, yoga, etc. Comparator: The control group 
did not implement intervention, and did not receive other hypolipidemic treatments. Outcomes: Primary outcomes: The primary outcomes are mainly evaluated by total cholesterol and triglycerides. Secondary outcome: The secondary outcomes are assessed by HDL-C, LDL-CI, ApoA1 and ApoB indexes.

Condition being studied: With the development of the social level and the improvement of living standards, people's dietary structure changes in the direction of high blood fat, high sugar and high fever, which leads to the occurrence of many diseases. Among them, hyperlipidemia refers to a situation where the mass metabolism disorder caused by various reasons increases blood cholesterol or/or triglyceride levels. Long-term increase in blood lipids can easily cause cholesterol to invade the walls of large blood vessels, deposit and accumulate, and promote the proliferation of smooth muscle cells and fibroblasts in the arterial intima, leading to coronary heart disease (CHD) and atherosclerosis (AS) and other cardiovascular and cerebrovascular diseases. Although drugs can treat hyperlipidemia, long-term medication will not only cause side effects, but also increase the economic burden. Studies have shown that aerobic exercise can affect the metabolism of blood lipids, as well as affect various indicators related to lipid metabolism.

\section{METHODS}

Search strategy: The plan searched terms are as follows: "high blood lipid" or "hyperlipidemia" or "cholesterol" or "triglyceride" or "HDL-C" or "LDL-C" and "aerobic exercises" or "aerobic fitness" or "walking" or "cycling" or "strength training" or "taiji" or "yoga".

Participant or population: Serum total cholesterol (TC) $\geq 5.20 \mathrm{mmol} / \mathrm{L}$, triglyceride (TG) $\geq 1.7 \mathrm{mmol} / \mathrm{L}$, high-density lipoprotein cholesterol (HDL-C) $\leq 1.04 \mathrm{mmol} / \mathrm{L}$, lowdensity lipoprotein cholesterol (LDL-C) $\geq$ $3.61 \mathrm{mmol} / \mathrm{L}$, no major disease, no blood lipid-lowering drugs in the past six months. All patients in the experimental group signed an informed consent form voluntarily to participate and were able to exercise as required.

Intervention: In the experimental group, all patients must perform aerobic exercises, including walking, cycling, strength training, taiji, yoga, etc. to improve TC, HDL-C, LDL-C and TG levels in patients with hyperlipidemia.

Comparator: The control group did not implement intervention, and did not receive other hypolipidemic treatments.

Study designs to be included: The inclusion criteria were randomized controlled trials, with aerobic exercise as the main form of intervention. The language of literature only includes Chinese or English.

Eligibility criteria: We will only include RCTs, whereas non-RCTs, and any other types of studies will be excluded.

Information sources: Electronic databases including Google Scholar, PubMed, Web of Science(WOS), the Cochrane Library, EMBASE and VIP Database for Chinese Technical Periodicals, China National Knowledge Infrastructure, and Wanfang. These databases will be searched to identify randomized controlled trials (RCTs) published January 1, 1980, and January 20, 2021. Language is limited with English and Chinese.

Main outcome(s): The primary outcomes are mainly evaluated by total cholesterol and triglycerides.

Additional outcome(s): The secondary outcomes are assessed by HDL-C, LDL-CI, ApoA1 and ApoB indexes.

Quality assessment / Risk of bias analysis: Two experienced authors(Zhao SP and Zhong J) will evaluate the methodological quality of randomized controlled trials according to the RCT quality evaluation criteria recommended in Cochrane Reviewer's Handbook 5.3.0. This 
assessment will consist of 9 items: generation of the random sequence, allocation concealment, blinding of participants and investigator, similar baseline, $=<15 \%$ dropouts, intention-totreat analysis, between-group comparison, point measure and measures of variability. The higher the score, the better the quality of the method. Any disagreements will be resolved via discussion with a third researcher (Zhang JP).

Strategy of data synthesis: We will use revman5.3 software for meta analysis. The relative risk (RR) with $95 \%$ confidence interval (CI) is used for dichotomous variables, and the weighted average difference (WMD) or standard mean deviation (SMD) with $95 \%$ confidence interval is used for continuous variables.

Subgroup analysis: We will conduct subgroup and meta-regression analysis to explore the sources of heterogeneity such as age, gender, disease course, intervention type, research quality, exercise intensity, frequency of use, and exercise time.

Sensitivity analysis: Sensitivity analysis will be performed to assess the reliability and robustness of the results. By changing the inclusion criteria, excluding low-quality studies, using different statistical analysis methods to observe changes in risk ratios (RR). If the document that has a significant impact on the combined $R R$ is excluded, the document is considered to be sensitive to the combined RR, otherwise it is not.

Country(ies) involved: China.

Keywords: aerobic Exercise, hyperlipidemia, TC, TG, systematic review, protocol, meta-analysis

Contributions of each author:

Author 1 - Shaoping Zhao - The author drafted the manuscript.

Email: zhaosp2623@163.com

Author 2 - Jiao Zhong - The author provided statistical expertise.

Email: 306463@whut.edu.cn
Author 3 - Caihong Sun - The author contributed to the development of the selection criteria, and the risk of bias assessment strategy.

Email: 249520239@qq.com

Author 4 - Junping Zhang - The author read, provided feedback and approved the final manuscript.

Email: 2291954641@qq.com 